\title{
Weiterbildung in der Corona-Krise
}

GERHARD BOSCH

\section{Vorläufiges Lagebild}

Die geplanten Transformationen in der deutschen Wirtschaft, wie die branchenweite Digitalisierung oder der Übergang vom Verbrennungs- zum Elektromotor in der Automobilindustrie, sind ohne Weiterbildung der Beschäftigten nicht möglich. Gleichzeitig aber hat der Lockdown infolge der Corona-Pandemie die Weiterbildung besonders hart getroffen. Das betrifft nicht nur die klassischen Formen der Weiterbildung in Präsenzveranstaltungen, sondern auch das informelle Lernen im Arbeitsprozess, das für viele Beschäftigte durch Kurzarbeit unterbrochen wurde.

Ein vollständiges Lagebild der Weiterbildung in der Covid-19-Krise ist gegenwärtig noch nicht möglich. Einige repräsentative Umfragen, wie die des Bundesinstituts für Berufsbildung (BIBB) und des Deutschen Instituts für Erwachsenenbildung (DIE) bei Weiterbildungsträgern oder Befragungen von Betrieben durch das Institut für Arbeitsmarkt- und Berufsforschung (IAB) und des Zentrums für europäische Wirtschaftsforschung (ZEW) wie auch die Geschäftsdaten der Bundesagentur für Arbeit (BA) zur arbeitsmarktpolitischen Weiterbildung ermöglichen jedoch einen ersten Überblick über die Gesamtsituation. Dabei zeigt sich auch: Sofern die Transformationen in den Unternehmen schon über große Investitionsvorhaben eingeleitet wurden, war ein Aufschub der dabei notwendigen Weiterbildungen nur unter erheblichen Folgekosten möglich. Wie die Betriebe mit diesem Problem umgegangen sind, wird künftige Forschung sicherlich genauer analysieren. Durch eine eigene, nicht repräsentative
Fallstudie bei Volkswagen Braunschweig kann ein Beispiel erfolgreicher Weiterbildung unter den schwierigen Umständen der Pandemie bei einem großen betrieblichen Transformationsprozess dargestellt werden. ${ }^{1}$

\section{Massive Einbrüche bei den Weiterbildungsanbietern}

Im sogenannten Weiterbildungsmonitor befragen BIBB und das DIE jährlich Träger der allgemeinen und beruflichen Weiterbildung $\mathrm{zu}$ wechselnden Schwerpunkten. Die letzte Befragung von 17411 Weiterbildungsträgern im Sommer 2020 konzentrierte sich auf die Auswirkungen der Covid-19-Krise. Der Geschäftsklimaindex, der schon seit Jahren in Anlehnung an den ifo-Index ermittelt wurde und das geometrische Mittel zwischen positiven und negativen Geschäftserwartungen der Weiterbildungsträger, gewichtet um das Dozentenstundenvolumen, ausdrückt, stürzte in der Krise bei allen Weiterbildungsanbietern auf ein Niveau noch unterhalb des Dienstleistungssektors ab. In allen Weiterbildungssegmenten - mit Ausnahme der durch die Bundesagentur und die Jobcenter finanzierten Weiterbildungsmaßnahmen - wurden Minuswerte gemessen. Der Einbruch war stärker als in der Finanzkrise 2008/09. Damals blieben die Werte überall positiv und erholten sich vor allem bei der durch betriebliche oder individuelle Beiträge finanzierten Weiterbildung schnell. ${ }^{2}$

Die befragten Weiterbildungsträger gaben an, im ersten Lockdown zwischen Mitte März und Mai $202020 \%$ aller Maßnahmen abgebrochen und $40 \%$ unterbrochen zu haben. Nur $3 \%$ wurden im geplanten Format wei-
1 Der Beitrag beruht auf einem Vortrag auf der gemeinsam von der Deutschen Vereinigung für sozialwissenschaftliche Arbeitsmarktforschung (SAMF), der Friedrich Ebert Stiftung (FES) und dem Wirtschafts- und Sozialwissenschaftlichen Institut (WSI) der Hans-Böckler-Stiftung durchgeführten Web-Konferenz vom 10. und 11. Juni 2021 zum Thema
„Die Bewältigung der Corona-Krise - Wie robust ist das deutsche Beschäftigungssystem?"

2 BIBB (Bundesinstitut für Berufsbildung) (2021): Datenreport zum Berufsbildungsbericht 2021, Bonn, S. 310. 


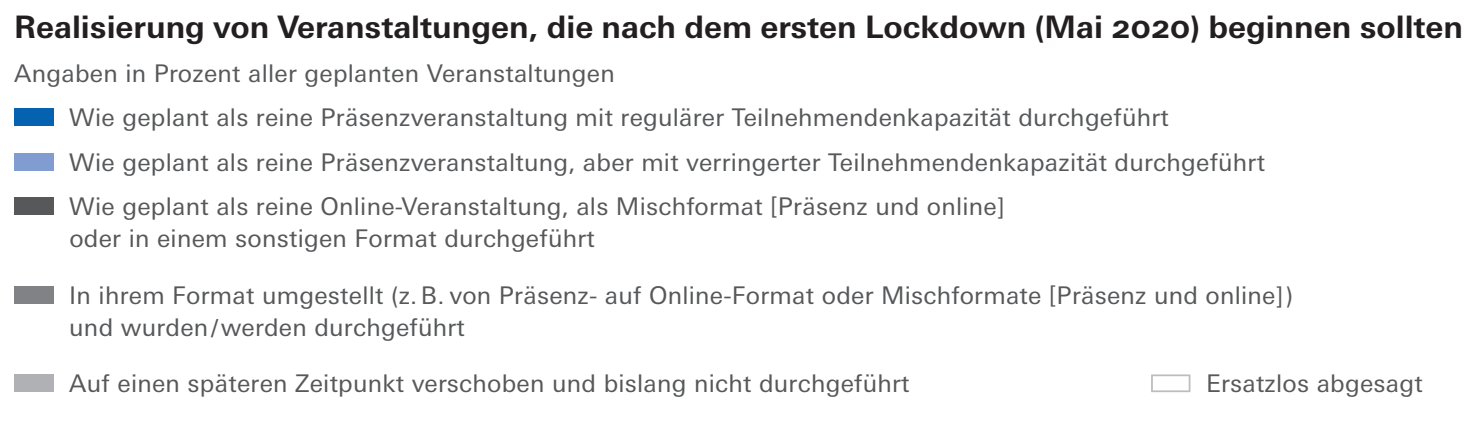

\begin{tabular}{r} 
Alle Anbieter \\
\hline Betriebliche Bildungseinrichtung \\
Berufliche Schule \\
Privat-gemeinnützig
\end{tabular}

tergeführt (Fernlehrgänge) und $37 \%$ wurden auf Online-Formate oder Mischformen umgestellt. Der Anteil der abgebrochenen bzw. unterbrochenen Kurse war am höchsten bei den Volkshochschulen (91\%), den Kirchen und Verbänden (73\%), den wirtschaftsnahen Einrichtungen (Kammern, Innungen) (63\%) und den betrieblichen Weiterbildungseinrichtungen (59\%), während vor allem (Fach-)Hochschulen und Akademien und berufliche Schulen von ihren offensichtlich guten technischen Infrastrukturen und ihren medienerfahrenen Teilnehmer*innen profitierten und mehr als zwei Drittel ihrer geplanten Maßnahmen auch realisierten. ${ }^{3}$

In der nächsten Phase nach dem ersten Lockdown wurden deutlich weniger Maßnahmen verschoben. Ebenso verringerten sich die Ausfälle sichtbar, da mit Hygienemaßnahmen wieder Präsenzkurse erlaubt waren. Rund $16 \%$ der Kurse wurden auf virtuelle Formate umgestellt (Abbildung 1). Auch hier waren die Berufsschulen und die (Fach-)Hochschulen und Akademien am erfolgreichsten, während die Volkshochschulen die größten Schwierigkeiten bei der Umstellung hatten. Dies liegt sicherlich nicht allein an der unzureichenden Infrastruktur der Volkshochschulen, sondern auch an deren potenziellem Teilnehmerkreis, der oft wenig lernerfahren und IT-affin ist (zum Beispiel in den Kursen zum Nachholen von Hauptschulabschlüssen oder den Integrations- und Sprachkursen für Migrant*innen). Zudem lassen sich freiwillig be- suchte Veranstaltungen in den Bereichen Kultur, Politik oder Gesundheit leichter absagen oder verschieben als betriebliche Maßnahmen, an denen die Umsetzung großer Investitionsprojekte hängt.

Die Ausfälle und auch die aufgrund der Hygienemaßnahmen verringerten Teilnehmer*innenzahlen führten zu erheblichen Umsatzeinbußen bei den Weiterbildungsträgern. Vier von zehn Anbietern nutzten Kurzarbeit. Zusätzlich wurden andere Hilfen genutzt, wie etwa die Soforthilfen für Selbstständige oder das SozialdienstleisterEinsatzgesetz, „auf dessen Grundlage antragsberechtigte Einrichtungen (z. B. Einrichtungen der Arbeitsförderung und von BAMF-Sprachkursen) Zuschüsse in Höhe von bis $\mathrm{zu} 75 \%$ ihrer durchschnittlichen Einnahmen erhalten können. “ ${ }^{4}$ Die Weiterbildungsinfrastruktur wurde mit den öffentlichen Hilfen zumindest kurzfristig weitgehend gesichert. Inwieweit sie sich durch einen kräftigen Aufschwung von Weiterbildungsaktivitäten, wie nach 2009, auch langfristig wieder erholt und stabilisiert, bleibt abzuwarten.

3 Christ, J./ Koscheck, S. (2021): Auswirkungen der CoronaPandemie auf Weiterbildungsanbieter: vorläufige Ergebnisse der wbmonitor Umfrage 2020. Version 1.0 BIBB Bonn, S. 3 .

4 BIBB (2021), a.a.O., S. 321. 
Befristete Verträge, die in diesem Sektor verbreitet sind, wurden in der Regel nicht verlängert und es kam auch zu Entlassungen. Da gerade der Weiterbildungssektor mit hohen Anteilen befristeter Honorarkräfte arbeitet, was schon vielfach kritisch analysiert wurde, ${ }^{5}$ waren die krisenbedingten Beschäftigungsrisiken in diesem Bereich offensichtlich höher als in Branchen mit höheren Anteilen unbefristeter Beschäftigung.

\section{Kurzarbeit kaum zur Weiterbildung genutzt}

Das Institut für Arbeitsmarkt- und Berufsforschung (IAB) befragt in seiner Sondererhebung „Betriebe in der Covid19-Krise“ 1500 Betriebe in regelmäßigen Abständen nach den Folgen der Pandemie. Nur 41\% der Betriebe gaben in der 14. Welle der Befragung (Juni/Juli 2021) an, über ausreichende Liquidität zu verfügen, ${ }^{6}$ was auf die prekäre Lage vieler Betriebe schließen lässt.

Die 5. Welle der Befragung (Oktober/November 2020) konzentrierte sich auf Weiterbildung und bestätigte die Ergebnisse des wbmonitors, dem zufolge im Lockdown viele geplante Weiterbildungsmaßnahmen abgesagt wurden. Als Gründe wurden vor allem die Kontaktbeschränkungen (96\% der Betriebe) genannt, die andere Faktoren wie beispielsweise die Unsicherheit über die Fortführung der Geschäftstätigkeit (28\%) oder zu hohe Kosten (15\%) überlagerten. Gefragt wurde auch nach dem Einsatz von E-Learning in der Krise. Immerhin $35 \%$ der Betriebe haben E-Learning in der Krise erstmalig eingesetzt und $44 \%$ haben diesen Einsatz ausgebaut. Das lässt auf einen Schub von E-Learning schließen, dessen Umfang allerdings nicht abgefragt wurde.

In der Finanzkrise 2008/09 wurden den Betrieben die Sozialversicherungsbeiträge für Kurzarbeiter nur erstattet, wenn mindestens die Hälfte der Kurzarbeit für Qualifizierungen genutzt wurde. Auf eine Wiederauflage dieser finanziellen Anreize für Weiterbildung wurde in der Covid-19-Krise zu Recht verzichtet, da diesmal durch die Kontaktsperren insbesondere auch personalintensive Dienstleistungsbetriebe mit geringen Liquiditätsreserven betroffen waren. Ohne die Erstattung der Sozialversicherungsbeiträge hätten diese Betriebe sicherlich viele ihrer Beschäftigten entlassen müssen.

Gleichwohl bieten sich Kurzarbeitsperioden für die Durchführung von Qualifizierungsmaßnahmen geradezu an, da die Bundesagentur für Arbeit unter bestimmten Voraussetzungen einen Teil der Kosten übernimmt und das neben den Kosten wichtigste Hindernis, nämlich die Freistellung der Beschäftigten im normalen Geschäftsbetrieb, entfällt. Auch Beschäftigte können von sich aus die freie Zeit für Weiterbildung einsetzen, falls keine anderen zeitlichen Verpflichtungen durch den Lockdown, etwa in der Kinderbetreuung, entstanden sind. Die IAB-Befragung ergab jedoch, dass nur rund jeder zehnte Betrieb mit Kurzarbeit die ausgefallene Arbeitszeit für Qualifizierung nutzte.

\section{ABBILDUNG 2}

Gründe, warum die Betriebe die durch Kurzarbeit ausgefallene Arbeitszeit nicht für Weiterbildung nutzten ${ }^{A}$ (Mehrfachnennungen)

Anteile in Prozent

Zeitpunkt Aufnahme Geschäftstätigkeit in vollem Umfang nicht absehbar Weiterbildung passt nicht in angepassten Arbeitsplan Geschäftliche Zukunft unsicher Thema Weiterbildung aktuell nachrangig Weiterbildungsangebot unpassend Kein Bedarf an Weiterbildung Ablehnung durch Beschäftigte

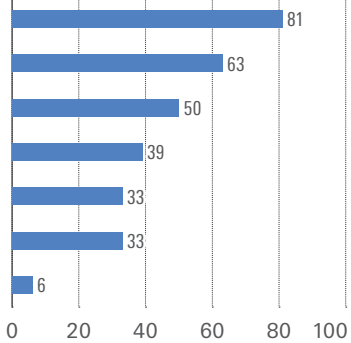

A Befragungsdaten von Oktober/November 2020

Quelle: Bellmann, L. et al. (2020): Weiterbildung in der Covid-19-Pandemie stellt viele Betriebe vor Schwierigkeiten, IAB Forum 9.12.2020, Nürnberg, S. 6

Die Unsicherheit über die Wiederaufnahme der geschäftlichen Tätigkeit, die Unsicherheit über die geschäftliche Zukunft und die Nachrangigkeit von Weiterbildung in der Krise wurden als die wichtigsten Gründe für die Weiterbildungsabstinenz in der Krise genannt (Abbildung 2). Dass Weiterbildung nicht in die angepassten Arbeitspläne passt, was $81 \%$ der Betriebe als Begründung angaben, scheint vorgeschoben, da die Betriebe die Arbeitspläne selbst gestaltet und darin ganz offensichtlich keine Weiterbildungszeit vorgesehen hatten.

Die Anteile der Beschäftigten in Weiterbildung und der Umfang der Maßnahmen wurden in der Betriebsbefragung des IAB nicht erhoben. Zahlen liegen hierzu aus einer Beschäftigtenbefragung des IAB zum Thema „Leben und Erwerbstätigkeit in Zeiten von Corona“ vor. Nach dieser Befragung nahmen nur $5 \%$ aller Kurzarbeiter ${ }^{*}$ innen während der Kurzarbeit an einer Weiterbildung teil. ${ }^{7}$

\section{Weniger berufliche Bildung in der Arbeitsmarktpolitik}

Wie schon in der Finanzkrise konnte über den massiven Einsatz von Kurzarbeit auch in dieser Krise Mas-

5 Dobischat, R. / Elias, A. / Rosendahl A. (Hrsg.) (2018): Das Personal in der Weiterbildung. Im Spannungsfeld von Professionsanspruch und Beschäftigungsrealität, Wiesbaden.

6 IAB (Institut für Arbeitsmarkt- und Berufsforschung) (2021): Ergebnisse aus Welle 14 der Studie „Betriebe in der Covid-19-Krise", 6. 7. 2021, Nürnberg.

7 Kruppe, T./ Osiander C. (2021): Kurzarbeit im Juni 2020: Rückgang auf sehr hohem Niveau, IAB Forum 23. 9. 2020, Nürnberg. 
senarbeitslosigkeit vermieden werden. Da diesmal aber auch der Dienstleistungssektor mit seinen vielen kleinen Unternehmen und oft hohen Anteilen befristet Beschäftigter betroffen war, konnte ein Personalabbau nicht ganz abgewendet werden. Die Zahl der Arbeitslosen stieg daher 2020 gegenüber dem Vorjahr um 428724 auf ein Gesamtniveau von 2695444 Personen an, während gleichzeitig aber in vielen Bereichen Fachkräfte fehlten. Übergänge in eine neue stabile Beschäftigung sind daher für viele Arbeitslose, vor allem die geringer Qualifizierten, nicht ohne eine berufliche Weiterbildung möglich.

Die Geschäftsstatistik der Bundesagentur belegt allerdings einen Rückgang der Eintritte in Weiterbildung 2020 im Vergleich zum Vorjahr. Der Rückgang lag bei den abschlussbezogenen Weiterbildungsmaßnahmen, die meistens länger dauern, erheblich über dem Durchschnitt. Weiterhin war der Rückgang im SGB II deutlich höher als im SGB III (Tabelle 1).

TABELLE 1

Eintritte in Maßnahmen der Förderung beruflicher Bildung 2019 und 2020

\begin{tabular}{|c|c|c|c|c|c|c|}
\hline & $\begin{array}{l}\text { alle } \\
\text { insgesamt }\end{array}$ & mit Abschluss & $\begin{array}{l}\text { SGB III } \\
\text { insgesamt }\end{array}$ & mit Abschluss & $\begin{array}{l}\text { SBG II } \\
\text { insgesamt }\end{array}$ & mit Abschluss \\
\hline 2019 & 324413 & 49666 & 204897 & 32989 & 119516 & 16677 \\
\hline 2020 & 267637 & 39716 & 181509 & 27298 & 86128 & 12419 \\
\hline Veränderung & $-17,5 \%$ & $-20,0 \%$ & $-11,4 \%$ & $-16,5 \%$ & $-27,9 \%$ & $-25,5 \%$ \\
\hline
\end{tabular}

Quelle: BA Statistik 2021

Aus diesen Daten kann man allerdings keinen Abschied von einer investiven Arbeitsmarktpolitik zugunsten einer schnellen Vermittlung - ähnlich wie bei den Rückgängen nach den Hartz-Gesetzen - ablesen. ${ }^{8}$ Ein Blick auf die monatlichen Zahlen zeigt einen starken Einbruch der Eintritte in Weiterbildungsmaßnahmen im April und Mai 2020 von deutlich über $50 \%$, einen langsamen Anstieg zum Jahresende 2020 hin mit überdurchschnittlichen Eintrittszahlen im September und Oktober 2020, die jedoch die Rückgänge im Lockdown nicht wettmachen konnten. Besonders hoch war der Rückgang mit fast $70 \%$ bei den Alleinerziehenden und den Arbeitslosen ohne beruflichen Abschluss. Zwar stieg auch bei diesen beiden Gruppen im Herbst 2020 die Teilnehmerzahl wieder an, ohne jedoch das Vorkrisenniveau zu erreichen.

Erste Gespräche mit der BA lassen erkennen, dass der Rückgang von Weiterbildungsinitiativen im April und Mai 2020 durch den Lockdown bei den Weiterbildungsträgern und nicht etwa durch eine veränderte Geschäftspolitik der BA und der Jobcenter verursacht wurde. Ein Grund mag auch in den Grenzen der virtuellen Weiterbildungs- beratung liegen. Virtuelle Formate können sicherlich alle erforderlichen Qualitäten bei der Information über Weiterbildungsangebote und die finanzielle Förderung bieten. Bei der Motivation hingegen haben sie unübersehbare Grenzen. Hinzu kommt, dass die Unsicherheit über die künftige Arbeitsmarktentwicklung auch die Arbeitslosen veranlasst hat, mit Weiterbildungsmaßnahmen zu warten.

Die besondere Betroffenheit der Alleinerziehenden hängt mit den Problemen der Kinderbetreuung in der Covid-19-Krise zusammen. Der überdurchschnittliche Rückgang bei den Arbeitslosen ohne beruflichen Abschluss lässt sich zum einen mit der hohen Bedeutung der persönlichen Beratung gerade für diese lernentwöhnte Gruppe begründen, was aufgrund der hohen Überschneidungen auch für die abschlussbezogenen Kurse gilt. Zum anderen war diese Gruppe vom Ausfall oder der Verschiebung der Kurse besonders betroffen, da sie meistens an Weiterbildungen teilnehmen, die eine physische Präsenz voraussetzen, wie zum Beispiel beim Schweißen oder dem Erwerb von Führerscheinen.

\section{Qualifizierung für die Transformation}

Gerade Ausgaben für Innovationen und Weiterbildung sind sehr stark prozyklisch. Zur Sicherung ihrer Zahlungsfähigkeit sparen die Unternehmen gezielt an den Ausgaben, die für das Tagesgeschäft nicht unabdingbar sind. Zudem sind Ungewissheiten über das künftige Geschäftsmodell häufig der Anlass, Innovationen zu verschieben. Gleichzeitig sinken aber die Opportunitätskosten für Innovationen, da es weniger Möglichkeiten gibt, über andere Aktivitäten Profite zu erzielen. Diese Krisenchancen kann man allerdings nur bei ausreichender Liquidität und klaren Zukunftsperspektiven nutzen.

Zwischen März und Juni 2020 befragte das ZEW Unternehmen nach den für 2020 geplanten Innovationsausgaben im Vergleich zum Vorjahr. Eine Besonderheit der Covid-19-Krise ist, dass der Rückgang dieser Ausgaben mit nur $2 \%$ deutlich geringer ist als in der Finanzkrise 2008/09 mit damals $11 \%$. Hinter diesen Durchschnittszahlen verbergen sich allerdings große Unterschiede. In den kleinen und mittleren Unternehmen waren die Rückgänge erheblich höher als in den großen. Das kann nicht überraschen, da ihre Kapitaldecke oft sehr dünn ist und ihre Geschäftsmodelle vielfach von den Aufträgen der großen Player abhängig sind. Auch die Branchenunterschiede sind markant. In der pharmazeutischen Industrie und der Biotechnologie nahmen aus naheliegenden Gründen die Innovationsausgaben zu.

In der Automobilindustrie mit ihrem schon begonnenen Umbau zur Elektromobilität blieben die Innovations-

8 Bosch, G. (2017): Weiterbildung und Arbeitsmarktpolitik: Qualifizierungsanforderungen - Paradigmenwechsel - Reformvorschläge, in: Soziale Sicherheit 66 (7-8), S. 261-268. 
ausgaben mit einer Abnahme von nur rd. $1 \%$ fast stabil. ${ }^{9}$ Das kann nicht verwundern, da sich ansonsten die ambitionierten Terminpläne der Transformation nicht einhalten ließen. Dies führt zu unserer Kurz-Fallstudie bei Volkswagen Braunschweig, die ein Beispiel für nicht aufschiebbare Transformationsweiterbildung in einem Unternehmen mit klaren Zukunftsplänen und ausreichender Liquidität ist. Am Braunschweiger Standort läuft die Fertigung von Kunststoffteilen und mechanischer Produkte aus. Gleichzeitig entstehen neue Arbeitsplätze im Bereich der Batteriesystemfertigung. Ohne eine Weiterbildung, die wegen des Umgangs mit Hochvoltaggregaten aus Sicherheitsgründen mit einer Prüfung abgeschlossen werden muss, ist eine Besetzung der neu entstehenden Arbeitsplätze im Betrieb nicht möglich. Eins-zu-eins-Versetzungen waren vielfach nicht durchführbar, da ein Teil der Beschäftigten für die Qualifizierung nicht infrage kam. Deshalb kam es zu Kettenversetzungen, von denen mehr Beschäftigte betroffen waren, als es rein rechnerisch zur Besetzung der neuen Arbeitsplätze der Fall gewesen wäre. Der Konzern stellte für die Weiterbildungsmaßnahmen im Konzern (andere Werke sind auch betroffen) ein Transformationsbudget für Weiterbildung in Höhe von 165 Mio. $€$ zur Verfügung. Die Standorte müssen sich um ein Weiterbildungsbudget aus diesem Topf bewerben. Erstattet werden die Kosten der Weiterbildung, nicht aber die Lohnausfallkosten, die jeder Betrieb selbst zu tragen hat. Jeder in Braunschweig betroffene Beschäftigte nahm an einer zweitägigen Fit-for-Change-Maßnahme teil, in der es um das „Lernen lernen“ ging. Es folgte eine selbstentwickelte 22-tägige Qualifizierung zur Elektrofachkraft. Die Qualifizierung folgte dem Hochlauf der Batteriemontage in den Jahren 2017 bis 2020. Die Weiterbildung für die Batteriefertigung wurde 2020 nicht unterbrochen, da sie als business essential eingeordnet wurde. Allerdings wurde das Hygienekonzept komplett überarbeitet (kleinere Gruppen, täglicher Selbsttest etc.). Es wurden eigene digitale Elemente, wie z.B. Erklärvideos entwickelt. Die Digitalisierung stieß allerdings an Grenzen, da das Unternehmen den Produktionsarbeitern anders als den Angestellten keine entsprechende Ausrüstung zur Verfügung gestellt hat.

\section{Fazit}

Die Covid-19-Krise führt zu erheblichen Einbrüchen in der Weiterbildung. Das informelle Lernen in der Arbeit entfiel in den oft sehr langen Kurzarbeitsperioden ersatzlos. Viele Weiterbildungskurse wurden gerade im ersten Lockdown 2020 und auch nach dessen Ende abgebrochen oder verschoben. Die Einbrüche waren besonders hoch bei Weiterbildungsträgern, die wenig Erfahrungen mit virtuellen Formaten hatten und für E-Learning nicht ausgerüstet waren. Besonders betroffen von diesem Einbruch waren Alleinerziehende aufgrund ihrer zusätzlichen Betreuungsverpflichtungen und gering Qualifizierte. Diese wenig lerngewohnte Gruppe ist besonders auf direkte persönliche Kommunikation und das Lernen in Gruppen angewiesen, ohne die oft weder die Motivation noch die Disziplin zum Lernen erzeugt werden kann. Weiterhin können zentrale Kompetenzen oft nur in realen Arbeitsprozessen über Beobachten, Imitation und Rückkoppelung durch erfahrene Ausbilder erworben werden.

Bemerkenswert in der Covid-19-Krise ist jedoch, dass zumindest die finanzkräftigen Großunternehmen ihre Innovationsausgaben weniger als in der Finanzkrise kürzten und offensichtlich auch die für schon eingeleitete Transformationsmaßnahmen notwendigen Weiterbildungen unter erschwerten Bedingungen (etwa durch die Hygienemaßnahmen) durchführten. Diese Erfahrung lässt sich allerdings vermutlich nicht verallgemeinern. Vor allem mittlere und kleine Unternehmen haben angesichts ihrer ungewissen Zukunft und erheblichen Liquiditätsengpässe Investitionen in Anlage und Weiterbildung zurückgestellt. Auch diese Krise ist infolge der negativen Auswirkungen auf Investitionen ein Innovationsbremser.

Allerdings wird die Pandemie in den nächsten Jahren einen Innovationsschub beim virtuellen Lernen auslösen. Positiv stimmt auch die rasche Zunahme der Weiterbildungseintritte in den beiden Rechtskreisen der Arbeitsmarktpolitik auf das Vorkrisenniveau. Durch die Soforthilfen scheint auch der Erhalt der Infrastruktur von Weiterbildungsträgern gesichert zu sein. Ob die Entlassung vieler Honorarkräfte zu Personalengpässen führen wird, bleibt abzuwarten.

\section{AUTOR}

GERHARD BOSCH, Prof. Dr., ist Senior Professor am Institut Arbeit und Qualifikation an der Universität Duisburg-Essen. Arbeitsschwerpunkte: Beschäftigung, Arbeitszeit, Berufliche Bildung, Löhne und soziale Sicherung im internationalen Vergleich.

gerhard.bosch@uni-due.de

9 Diekhof, J./ Krieger, B. / Licht, G. / Rammer, C. / Schmitt, J./ Stenke, G. (2021): The Impact of the Covid-19 Crisis on Innovation: First In-Sights from the German Business Sector, ZEW - Leibniz-Zentrum für europäische Wirtschaftsforschung: ZEW Expert Brief 21-06, Mannheim. 